\title{
Assessment of airborne and spaceborne thermal infrared remote sensing for detecting and characterizing landfills
}

\author{
B. Beaumont, J. Radoux \& P. Defourny \\ Earth and Life Institute, Université catholique de Louvain, Belgium
}

\begin{abstract}
This work deals with the application of thermal infrared remote sensing for landfill management. It aims to address two research questions: (i) Can we detect all past and present landfills using spaceborne data archive, and (ii) is airborne remote sensing an efficient tool for landfill site characterization? The results extracted from the spaceborne data analysis show that the detection of landfills is possible during their activity phase. The importance of the context of acquisition is highlighted by the application of the developed detection methods for distinct geographical contexts. High spatial resolution airborne data acquired at dawn proves to be a relevant information source for the characterization of thermal anomalies present on multiple landfills.

Keywords: landfill detection, landfill characterization, thermal infrared remote sensing, ASTER, Landsat, airborne FLIR thermal data set, CETeM.
\end{abstract}

\section{Introduction}

Sustainable waste management is one of the most important environmental concerns in today's society. Globally, the burial of waste in landfills remains the most common management method despite the development of alternative technologies (Frandegard et al. [1]). Such an approach is problematic due to the fact that resources that are buried, containing both energy and materials, are wasted. In addition, this practice causes many environmental and health issues that require a continuous management. The restoration of landfills as part of this management, commonly referred to as landfill mining, also offers many opportunities (energy production, land and resources recoveries).

The MINERVE project was launched in this context. A three axes methodology was developed with the aim of shortening the life cycle of waste: (i) characterization, (ii) mineralization and (iii) landfill mining. The contribution 
of the Environmetrics and Geomatics lab of the UCL (Université catholique de Louvain) to this project is to evaluate the potential of remote sensing in the waste management field, especially with the goals of landfills detection and characterization. Indeed, the location of landfills remains unknown in many parts of the world. Given the dispersion of these sites, a field survey of a large area would prove too costly. In addition, many sites are illegal, old and forgotten. They are therefore not included in the current databases. In the context of covering a large territory, the use of spaceborne and airborne remote sensing technologies has proved its efficiency in many current environmental issues. Nevertheless, the application of these technologies for waste management is still limited to specific objectives.

Remotely sensed data are often used for landfill sites mapping and as decision support tools for the implementation of new sites. Ottavianelli et al. [2] reviewed the majority of papers dealing with such uses until 2005. They conclude that spaceborne remote sensing had not yet proved to be a valid tool for an accurate quantitative analysis of landfills and that it can only support ground remediation efforts based on the expertise of a visual interpreter and the knowledge of the landfill operator. Since then, Silvestri and Omri [3] used image classification techniques on high resolution IKONOS images and Geographical Information System (GIS) to identify uncontrolled landfills at regional scale. Biotto et al. [4] deepened the previous study by proceeding to a GIS statistical analysis in order to produce a probability map narrowing down the set of possibly contaminated sites detected.

According to the literature, use of the Thermal InfraRed (TIR) spectral range appears promising for the detection and characterization of landfills by remote sensing. Indeed, one assumption that can be made is that the biological activity occurring inside these sites (producing gas and heat up to $333 \mathrm{~K}$, CWBI [5]) generates streams of visible surface heat. As a consequence, landfills should theoretically address surface thermal anomalies that offer the potential to distinguish them from their surroundings on TIR images and, depending on the spatial resolution of the data, allow the characterization of the biological activities occurring inside the various sectors of them. Data acquisition in the TIR range can be performed inside two spectral windows: MWIR (Medium Wavelength InfraRed [3.8-4.2 $\mu \mathrm{m}]$ ) and LWIR (Long Wavelength InfraRed [8-12 $\mu \mathrm{m}]$ ) (MIVIM [6]). Due to the terrestrial objects emission peaks and the constant Land Surface Emissivity (LSE) values observed in the LWIR subdomain, this spectral window is the most commonly used.

Regarding TIR spaceborne acquisition and the required spatial resolution of the data, only images obtained through the series of Landsat (Landsat-5 $(120 \mathrm{~m})$, Landsat-7 $(60 \mathrm{~m})$ and Landsat-8 $(60 \mathrm{~m})$ ) and ASTER (Advanced Spaceborne Thermal Emission and Reflection; $90 \mathrm{~m}$ ) satellites are relevant for waste management studies. A few papers, [7-9] focused on the use of Landsat TIR data for sites characterization and for the detection of suspicious dumping areas. These authors proved that the difference in LST between active landfills (positive thermal anomalies) and their surroundings is correlated to some extent with the speed and extent of biodegradation in the landfilled material and the 
release of landfill gases. However, they highlighted that the thermal inertia of bare soil during the day, often characterizing landfill surfaces, can increase the contrast of temperature observed. The spatial resolutions proposed by the Landsat TIR sensors were also not sufficient to study the correlation between biogas fluxes in gas extraction wells and the LST observed.

Regarding TIR airborne acquisition, higher spatial resolution and more precise radiometric resolutions can be obtained. However, unlike the free access to Landsat and ASTER spaceborne data, airborne acquisition require the planning of a flight with a private operator, and are therefore expensive. Creating time series of such data appears thus less affordable. The potential of airborne TIR data has then been assessed in a limited number of studies mainly focused on single image acquisition. The main results expressed by Hedges et al. [10] and Diot et al. [11] were that the positive thermal anomalies detected on landfills were only partially related to gas flows (surface characteristics such as water areas and lighting systems induced false detection). Solar radiation impacted negatively the analysis and data acquisition at dawn is therefore advised. The combined use of spaceborne and airborne thermal imaging for illegal dumping sites detection and characterization has been recently assessed by Persechino et al. [12]. They proved, at one hand, that airborne high spatial resolution thermography is ideal for the detection of environmental contamination such as weakness (high biogas emissions) and risk areas (fires or explosions) and, on the other hand, that the combined use of airborne and spaceborne remote sensing tools allows to have overviews of an area at different scales improving the diagnosis of illegal dumping sites.

Finally, the comparison between thermal anomalies detected by TIR data acquired at ground level and biogas fluxes measurements studied by Raco et al. [13] shows that these two sources of data are only partially correlated but that they provide additional information about the state of the site. A positive thermal anomaly may come from various conditions such as higher thermal production beneath the ground or higher thermal conductivity of the surface. Given the effectiveness of the coverage and the gas collecting systems, high gas fluxes may be detected in spite of low superficial temperature due to an inappropriate collecting systems, as well as high thermal anomalies may produce no gas fluxes, thanks to an efficient coverage system.

The aim of this paper is to assess the potential of spaceborne and airborne TIR remote sensing data in the waste management field. More precisely, the first objective is to develop a methodology for allowing locating biologically active landfills on a territory. The second goal is to study the capacity of high resolution TIR data for the characterization of landfills in term of biological activity related to the organic waste decomposition process.

\section{Materials and methods}

\subsection{Study areas}

Exhaustive analysis has first been performed on recent and old landfills located near Louvain-la-Neuve in Belgium. The characterization study is focused on the CETeM (Centre d'Enfouissement Technique de Mont-St-Guibert; 44.3 ha wide). 
This site presents a wide range of different contexts (still in activity, recently capped and old areas covered with vegetation) and it appears therefore to be ideal for assessing the potential of remote sensing in this objective. The generalization of the detection results has then been tested on Tunisian landfills thanks to the experience of the CWBI [5]. The two study areas are visible at fig. 1 .

\subsection{Data sets}

Two spaceborne (Landsat and ASTER) and one airborne data sets were used in the LST analyses carried on the Belgian study area (fig. 1). Validation was done using several sources (Google Earth archive, Orthophotos acquired by the Walloon Region [14] and RapidEye images). The land cover classification (COSW) was produced by the Walloon Region [14]. The census of landfill sites present in this study area was provided by the SPAQuE [15]. Ground data measurements on the CETeM were provided by Shanks [16] and ISSeP [17].

Only Landsat images were used for the detection of landfills over Tunisia (fig. 1). The validation data available was the Google Earth images archive.

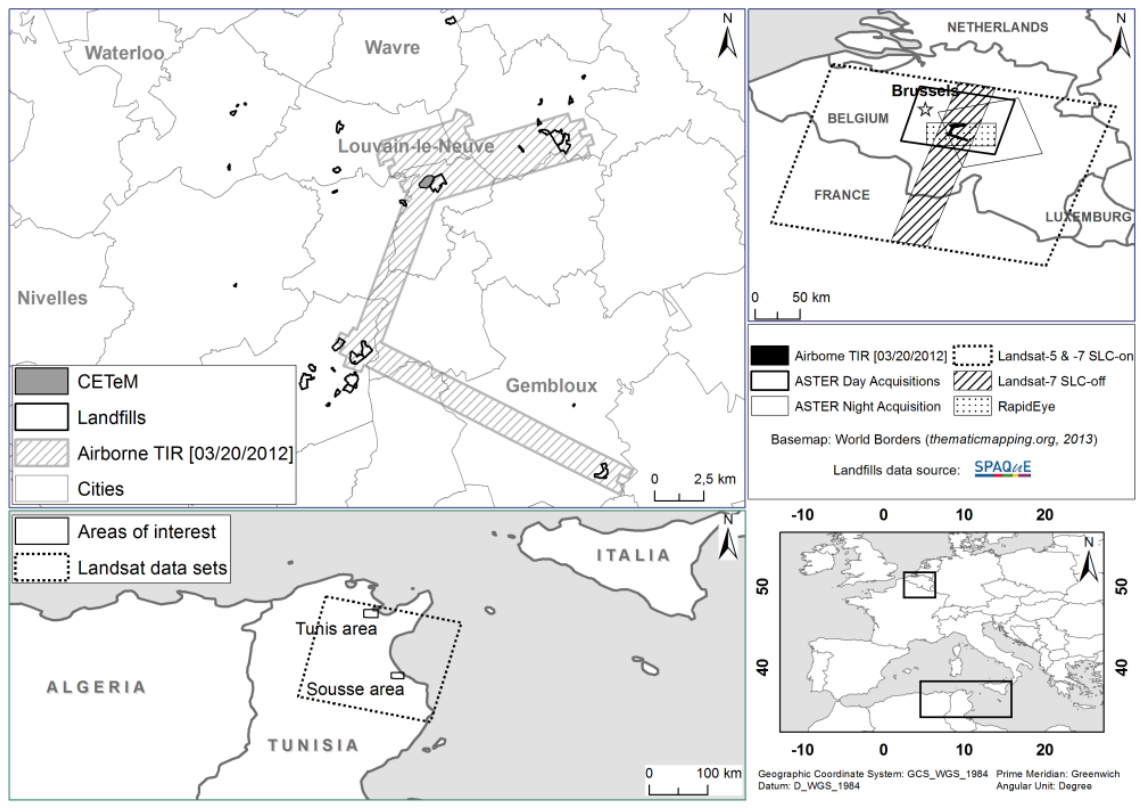

Figure 1: $\quad$ Belgian and Tunisian study areas and corresponding data sets.

\subsubsection{Landsat-5 TM \& Landsat-7 ETM+ data sets}

The Landsat data sets used in this study consist in cloud-free Landsat-5 images with Thematic Mapper (TM) sensor (from 1986 to 2010; 6 images over Belgium and 24 over Tunisia) and Landsat-7 images with Enhanced Thematic Mapper Plus (ETM+) sensor (from 1999 to 2012; 22 images over Belgium and 10 over 
Tunisia). The acquisition time is around 11:20 am local time. These satellites offer, in addition to the visible and near infrared bands traditionally met, data acquisition in the LWIR range [10.4-12.5 $\mu \mathrm{m}$ ]. Thermal data since $02 / 25 / 2010$ is re-sampled to $30 \mathrm{~m}$ as to match the spatial resolution of the other multispectral bands of Landsat satellite (USGS [18]). On May 31, 2003, the Scan Line Corrector (SLC), which compensates for the forward motion of Landsat-7, failed. Since then, useful image data can only be acquired in the central part of the scene ( $22 \mathrm{~km}$ wide). Landsat scenes acquired in this study were preprocessed at L1T level. Geometric correction and radiometric calibration of the sensors were thus already performed. The conversion of DN from thermal bands into LST requires a multistep process. The general procedure to obtain effective temperature at the sensor is fully described in Chander et al. [19]. The NDVI (Normalized Difference Vegetation Index) Thresholds Method implemented by Copertino et al. [20] has then been used for the conversion of $\mathrm{T}_{\mathrm{B}}$ into LST.

\subsubsection{ASTER data set}

ASTER satellite data offer a spectral ranging from the visible to the infrared part of the electromagnetic spectrum since 2000. Five thermal channels in the LWIR range [8.125-11.65 $\mu \mathrm{m}$ ] are available. Unlike Landsat data that are acquired only during the day, ASTER data can be acquired day and night. The archive products list is however much more limited. 12 ASTER cloud-free products AST_08; 11 day (around 11:50am local time) and 1 night (around 10:30pm local time) products, providing LST data, were available for the Belgian study area (from 2003 to 2011). Orthorectification has been performed using ERRUISSOL DEM (Digital Elevation Model; SPW [15]) in ENVI 4.8.

\subsubsection{Airborne data set}

The airborne data set was acquired between 3:24 and 4:40 am local time on March 20, 2012. The sensor is a FLIR SC7600-BB operating in the MWIR range [3.5-6 $\mu \mathrm{m}]$. Absolute precision of the brightness temperature (TB) measured is about $1 \mathrm{~K}$ after calibration (FLIR [21]). Climatic conditions during the flight were, according to Vaughan et al. [22], close to be optimal (ground air temperature of $275 \mathrm{~K}$, perfect visibility and slow winds; Shanks [16]). Such weather should emphasize the contrast between ground temperature heated by biological activities happening in the landfills and other surfaces. Radiometric calibration of the data was provided by the flight operator (Aerodata [23]). Given the spatial resolution of the data $(0.5 \mathrm{~m})$, the heterogeneity of the LSE in the MWIR range and the uncertainty of it in relationship with the various ground materials, it was decided to work with an emissivity parameter equal to 1 for this data set $($ LST $=$ TB). Such a simplified approach is often used in the literature (e.g. EUROSENSE [24]). Geometric corrections of the data were performed using ERDAS Leica Photogrammetry Suite software.

\subsection{Methodology}

The suitability of airborne and spaceborne remote sensing for landfills detection was firstly assessed. The methodology developed for the analysis of the satellite time series aimed to define the optimal context of acquisition for the detection of 
landfills. Single image analysis was then first carried. The averaging of the signal on multiple images by periods or acquisition contexts was then tested, if needed, in order to improve the discrimination of landfills from the other land use/land cover classes. The potential for landfills characterization was secondly assessed using the high resolution TIR airborne data set.

\section{Results}

\subsection{Detection of landfills}

\subsubsection{Landsat-5 TM and Landsat-7 ETM+ data sets}

The image-by-image analysis of LST data extracted from the Landsat time series has been conducted in order to determine the context of acquisition which allows the best detection of landfills. Regarding the Belgian study area, summer acquisitions (especially in July; fig. 2) give the best results. The explanation comes from the observed negative correlation between NDVI (standardized index of greenness (relative biomass) with NDVI $<0.2=$ bare ground and NDVI $>0.5=$ dense green vegetation; Yue et al. [25]) and LST that peaked during the summer months. Bare ground surfaces (such as landfills in activity, harvested agricultural fields) and urban areas show higher LST profiles (2-10 K higher) than other land cover classes. A masking of the urban areas (requirement illustrated at fig. 4) is then needed in order to detect landfills. It can be easily achieved based on ancillary data when they are available (in this case, COSW data). During the summer period, the amount of harvested fields with bare soil cover is limited compared to other times of the year. The number of areas with high LST patterns, similar as the one observed on landfills, is therefore reduced.

The use of LST values averaged over multi-temporal data acquired during the optimal context of acquisition defined in fig. 2 (acquisitions of 07/14/2005 and 07/01/2006) reduces the number of false detection and brings out areas with

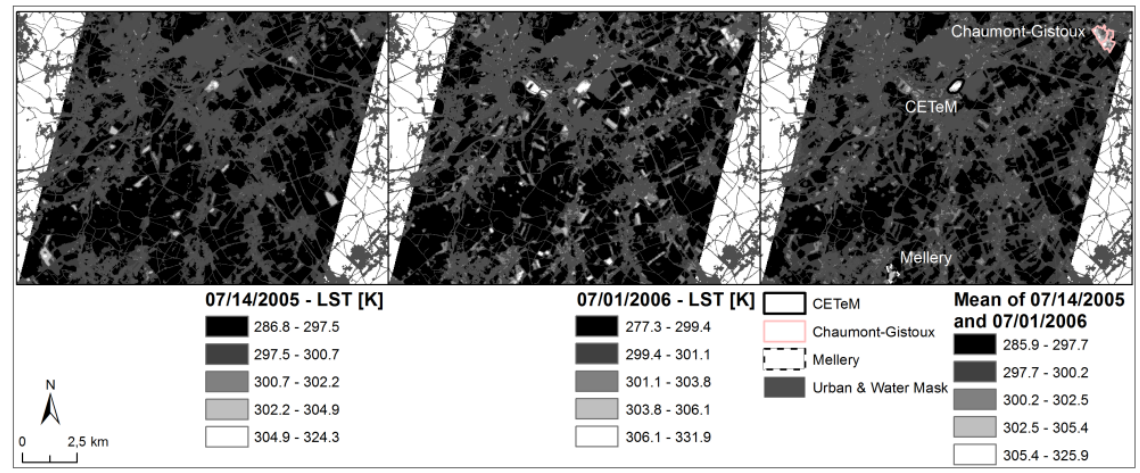

Figure 2: Detection of landfills using LST extracted from Landsat-7 images from [07/14/2005] and [07/01/2006] and by averaging these two data. 
consistent high temperature profiles (such as the CETeM and, in a lesser extent, other landfills such as a rehabilitated one (Mellery discharge) and one on the north-east part of the study area (Chaumont-Gistoux discharge)). The LST of agricultural fields, which present high variability in surface covers from year to year, are thus limited. From 15+ suspected sites detected on 07/14/2005 and $07 / 01 / 2006$ images (size of the area about $200 \mathrm{~km}^{2}$ ), we reduce the numbers of suspected sites to 4 . The validation of these sites using other remotely sensed data reveals that 3 of them are landfills, the last one being an agricultural field.

The CETeM is a landfill which was still in activity in the years 2000's. This explains the better results found for this site on these acquisitions. The use of older data allows a better discrimination of former landfills. However, given the spatial resolution of Landsat data and the lower biological activity occurring in small size sites, the detection of small landfills has not been satisfying. The uncertainty about the type of waste buried in these sites (organic or inorganic waste?) doesn't allow demonstrating the ability of TIR data for their detection.
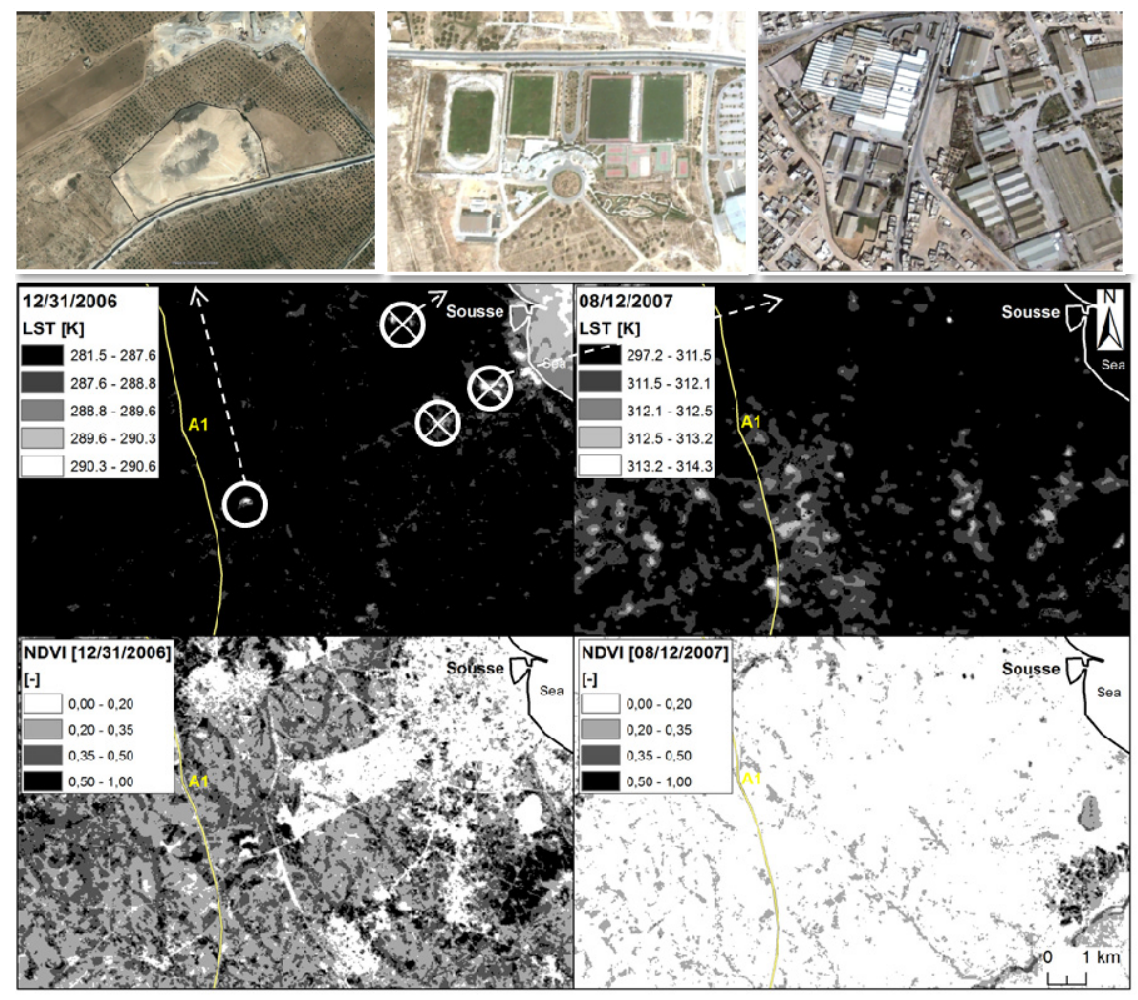

Figure 3: Landfill detection analysis over the Sousse area (Tunisia) using Landsat images. Comparison of the detection potential between green (12/31/2006) and dry conditions (08/12/2007) using derived LST data and NDVI. Validation bases on Google Earth archive images. 
Regarding the Tunisian study area, the single-image analysis allows the definition of the optimal context of acquisition, here related to the presence of green vegetation. Unfortunately, such situation is rare on these regions and finding it during the activity phase of the landfills to be detected is thus not guaranteed. In our study case, only one acquisition showing a pronounced green vegetation cover was found on $12 / 31 / 2006$. The comparison between this acquisition and another taken in a dry context (08/12/2007), visible at fig. 3, shows the highest separability potential on the winter acquisition. In this case, the majority of urban areas don't show high LST profiles (due to different construction materials and climatic context). However, some false detections still occur between landfills and urban areas. Given their small amount (only 4 sites detected), the validation procedure of these sites can be easily achieved and allow the identification of the only one biologicaly active landfill present in this area in 2006.

\subsubsection{ASTER data set}

Similar results as the one cited during the Landsat analysis were obtained for the assessment of the AST_08 product. Fig. 4 illustrates the correlation between the LST values extracted from both products acquired on $07 / 17 / 2006$. A R $\mathrm{R}^{2}$ of 0.81 was found between them (on 100 sample points). The lower spatial resolution of ASTER TIR data as well as the relatively young age of the sensor compared to Landsat-7 reduces the usefulness of these data for landfills detection.
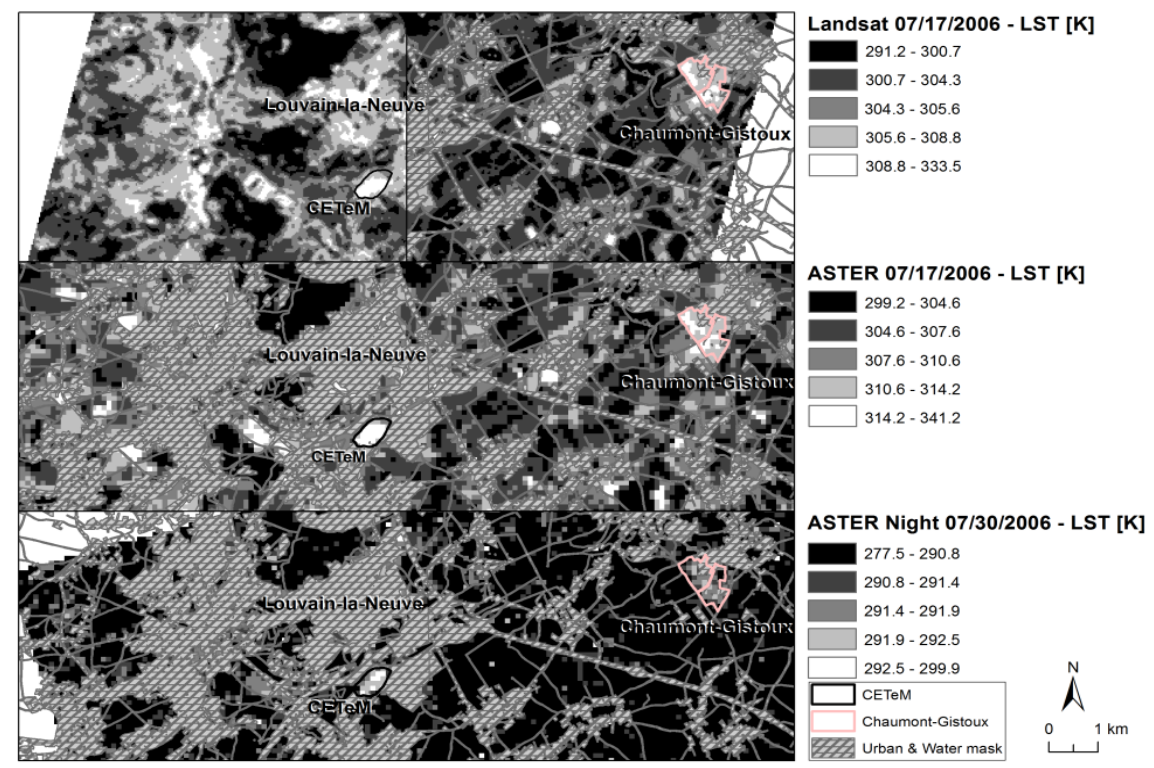

Figure 4: Comparative analyses between Landsat-7 ETM+ LST image from 07/17/2006 and ASTER AST_08 LST products from 07/17/2006 and 07/30/2006 (nocturnal acquisition). Illustration on 07/17/2006 image of the requirement of an urban areas mask. 
The acquisition of 07/30/2006 (fig. 4), acquired during night time, presents the theoretical interest to overcome the incident solar radiation effects. Given the acquisition time at nightfall (10:30 pm local time), these effects and their impacts on the warming of surface (thermal inertia) are still present. Great potential is nonetheless seen with the two landfills still in activity (CETeM and Chaumont-Gistoux) showing higher LST values than their surroundings (2-4 K higher). The observed results are probably improved due to greater warming of the bare soils characterizing these sites in opposition to the other land cover classes.

\subsubsection{Airborne data set}

Due to its acquisition period and its high spatial resolution, the suitability of the airborne MWIR data set for the detection of landfills against the other land cover classes was not demonstrated (fig. 5). In winter, lots of surfaces are characterized by small vegetation covers such as the ones found on landfills. The distributions of LST data extracted for these areas present a high correlation with those from landfills. The detection can thus not be achieved, even with a mask of the urban and water areas (high LST), through the analysis of this data set.

\subsection{Characterization of landfills}

The potential of nocturnal MWIR data acquisition for multiple landfill sites characterization is illustrated on the CETeM in fig. 5. This figure highlights the possibility of diagnosing weakness areas (higher biogas leaks resulting in higher

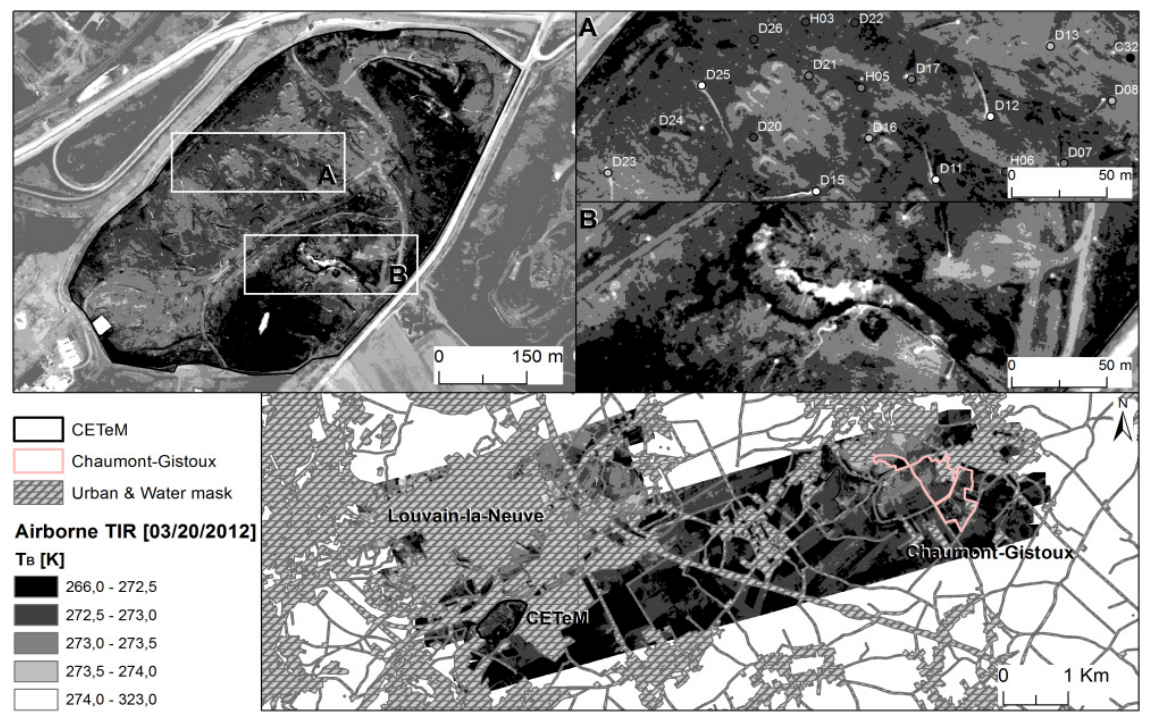

Figure 5: Characterization and detection of landfills using $\mathrm{T}_{\mathrm{B}}$ derived from the airborne MWIR [03/20/2012] data set. Top image: Characterization of the CETeM with (A) infiltration strips and gas extraction wells and (B) weakness areas. Lower image: Detection of landfills analysis with a mask of urban and water areas. 
LST (0.5-4 K higher)) as well as the majority of landfills' infrastructure (degasification wells, collector tubes; subfigure A).

The weakness areas are preferentially found around gas extraction wells and in areas with pronounced relief or recently modified (subfigure B). The delineation of these areas indicates that the capping of the landfill and/or the gas extraction wells should be controlled. The validation of the weakness area detected has been performed using gas fluxes measurements made with an Inspectra laser a few months later (ISSeP [17]). Currently, existing data could not quantitatively validate the results because of the high temporal variability of biogas emissions and the impact of surface characteristics on LST measurements (due to LSE variation).

The range of the observed thermal response is greater on the CETeM than on the other landfills. This dynamic, which is partly explained by the age, the quantity of waste buried and by the stage of rehabilitation of the site must also be linked with the diversity of surfaces, and the LSE that characterizes them. This diversity is much more pronounced on the CETeM which is partially still in activity.

\section{Discussion}

The first objective was to assess the suitability of spaceborne TIR time series for landfills detection at regional scale. A two-step methodology was developed including single image analysis and multi-temporal signal averaging. To the best

of our knowledge this approach has never been employed before in this aim. Considering the results obtained, the combined use of LST data with high resolution optical data and GIS (as a new parameter to the methodology developed by Biotto et al. [4]) seems to be a complete solution for a precise detection of landfills.

Some of the detected landfills were former sites not anymore active (currently rehabilitated or covered by dense vegetation). Their detections at present time were thus not possible. This fact highlights the importance of satellite data archive. A visit to the USGS Landsat Global Archive web page [26] allow estimating the amount of Landsat data that an operator can expect to apply the landfills detection process in its area of intervention. The TIR data availability is much more important over the United States. The global coverage is relatively new (since 1999 and the Landsat-7 launch) and archive data availability appears thus to be the main limitation for old sites detection.

The suitability of high spatial resolution airborne TIR data acquired at dawn for multiple landfill sites characterization has secondly been assessed. Even if some positive thermal anomalies can be detected on the landfills studied, their validity as weakness areas could not be demonstrated. Given our results, the main conclusions expressed by Raco et al. [13] still appear to be valid. As to take advantage of the main benefits of airborne remote sensing (rapid acquisition over multiple sites without on-sites interventions), sensors specifically oriented towards the detection of biogas emitted by landfills should be developed. 


\section{Conclusion}

The monitoring of landfills using spaceborne and airborne remote sensing technologies has been assessed. On the one hand, the use of satellite data helps to detect and delineate landfills at regional scales. The open access to the global archive allows the detection of landfills that are now forgotten and helps to understand the dynamics that occurred in and around these sites over time. On the other hand, high spatial resolution airborne TIR remote sensing proves to be a useful tool for monitoring a large number of sites in a short period of time.

The recent and future advances in terms of sensors (Landsat- 8 TIRS, HyperCam) and vectors (UAV) need to be highlighted. Firstly, the continuity of global satellite TIR data acquisitions is guaranteed. The potential of still in activity and future landfill sites detection is thus enhanced. Secondly, these advances allow performing faster and more precise acquisition over specific sites in order to detect thermal anomalies and biogas fluxes.

\section{Acknowledgements}

The authors would like to thank all the members of the MINERVE projects as well as GreenWin for the funding of this project through the Plan Marshall 2.vert.

\section{References}

[1] Frandegard, P., Krook, J., Svensson, N. \& Eklund, M., A novel approach for environmental evaluation of landfill mining. Journal of Cleaner Production, pp. 1-11, 2012.

[2] Ottavianelli, G., Hobbs, S., Smith, R. \& Bruno, D., Assessment of hyper spectral and SAR remote sensing for solid waste landfill management. Space Research Centre, School of Enfineering, Cranfield University, 2005.

[3] Silvestri, S. \& Omri, M., A method for the remote sensing identification of uncontrolled landfills: formulation and validation. International Journal of Remote Sensing, 29:4, pp. 975-989, 2008.

[4] Biotto, G., Silvestri, S., Gobbo, L., Furlan, E., Valenti, S. \& Rosseli, R., GIS, multi-criteria and multi-factor spatial analysis for the probability assessment of the existence of illegal landfills. International Journal of Geographical Information Science, Vol. 23, No. 10, pp. 1233-1244, 2009.

[5] CWBI, http://cwbi.fsagx.ac.be/

[6] MIVIM, http://www.mivim.gel.ulaval.ca/

[7] Kwarteng, A.Y. \& Al-Enezi, A., Assessment of Kuwait's Al-Qurain landfill using remote sensed data. Journal of Environment Science and Health, 39(2), pp. 351-364, 2004.

[8] Faisal, K., Environmental monitoring of landfill sites using multi-temporal remote sensing images. Ryerson University, pp. 1-78, 2011. 
[9] Shaker, A. \& Yan, W.Y., Trail road landfill site monitoring using multitemporal Landsat satellite data. Canadian Geomatics Conference 2010 and ISPRS COM I Symposium, Calgary, Canada, 2010.

[10] Hedges, P.D., Ellis, R.J. \& Elgy, J., Airborne remote sensing as a tool for monitoring landfill sites within an urban environment. Sampling Environmental Media, ASTM STP 1282, James Howard Morgan, Ed., American Society for Testing and Materials, pp. 63-77, 1996.

[11] Diot, M., le Golvan, Y.M., Bogner, J., Burkhalter, R., Chanton, J., Tregourès, A. \& Schaegis, L., Landfill biogas emission measurements: Qualification and quantification of the pathways of emitted methane. Intercontinental Landfill Research Symposium, Luleå, Sweden, 2000.

[12] Persechino, G., Schiano, P., Lega, M., Napoli, R.M.A., Ferrara, C. \& Kosmatka, J., Aerospace-based support systems and interoperability: the solution to fight illegal dumping. Waste Management, pp. 3-11, 2011.

[13] Raco, B., Scozzari, A., Guidi, M., Lelli, M. \& Lippo, G., Comparison of two non-invasive methodologies to monitor diffuse biogas emissions from MSW landfills soil: A case study. Proceedings Sardinia 2005, Tenth International Waste Management and Landfill Symposium, Cagliari, Italy, 2005.

[14] SPW, http://www.wallonie.be/

[15] SPAQuE, http://www.spaque.be/

[16] Shanks, http://www.shanks.be/

[17] ISSeP, http://www.issep.be/

[18] USGS, http://www.usgs.gov/

[19] Chander, G., Markham, B. \& Helder, D., Summary of current calibration coefficients for Landsat MSS, TM, ETM+ and EO-1 ALI sensors. NASA, 2009.

[20] Copertino, V.A., Di Pierro, M., Scavone, G. \& Telesca, V., Comparaison of algorithms to retrieve LST from Landsat-7 ETM+ IR data in the Basilicata Ionian band. Tethys, 9, pp. 25-34, 2012.

[21] FLIR, http://www.flir.com/

[22] Vaughan, R.G., Keszthelyi, L.P., Lowenstern, J.B., Jaworowski, C. \& Heasler, H., Use of ASTER and MODIS thermal infrared data to quantify heat flow change at Yellowstone National Park. Journal of Volcanology and Geothermal Research, pp. 72-89, 2012.

[23] Aerodata, http://aerodata-surveys.com/

[24] EUROSENSE, http://www.eurosense.com/

[25] Yue, W., Xu, J., Tan, W. \& Xu, L., The relationship between Land NDVI with remote sensing: application to Shanghai Landsat 7 ETM+ data. International Journal of Remote Sensing, 28:15, pp. 3205-3226, 2007.

[26] USGS, http://landsat.usgs.gov/documents/StateOfTheArchive_web.pdf 